\title{
Stage II Lip and Oral Cavity Cancer AJCC v8
}

National Cancer Institute

\section{Source}

National Cancer Institute. Stage II Lip and Oral Cavity Cancer AJCC v8. NCI Thesaurus.

Code C132730.

Stage II includes: T2, N0, M0. T2: Tumor size $2 \mathrm{~cm}$ or less in greatest dimension, DOI greater than $5 \mathrm{~mm}$ and equal or less than $10 \mathrm{~mm}$ or tumor greater than $2 \mathrm{~cm}$ but $4 \mathrm{~cm}$ or less in greatest dimension and $10 \mathrm{~mm}$ or less DOI. N0: No regional lymph node metastasis. M0: No distant metastasis. (AJCC 8th Ed.) 